\title{
Papers
}

\section{Three day versus five day treatment with amoxicillin for non-severe pneumonia in young children: a multicentre randomised controlled trial}

\author{
ISCAP Study Group
}

\begin{abstract}
Objective To assess the efficacy of three days versus five days of treatment with oral amoxicillin for curing non-severe pneumonia in children.

Design Randomised, double blind, placebo controlled multicentre trial.

Setting Outpatient departments of seven referral hospitals in India.

Participants 2188 children aged 2-59 months, 1095 given three days of treatment and 1093 given five days.

Intervention Oral amoxicillin 31-54 mg/ $\mathrm{kg}$ /day in three divided doses.

Main outcome measures Treatment failure: defined as development of chest indrawing, convulsions, drowsiness, or inability to drink at any time; respiratory rate above age specific cut points on day 3 or later; or oxygen saturation by pulse oximetry $<90 \%$ on day 3 .

Results The clinical cure rates with three days and five days of treatment were $89.5 \%$ and $89.9 \%$, respectively (absolute difference $0.4(95 \%$ confidence interval -2.1 to 3.0$)$ ).

Adherence to treatment regimen was $94 \%$ and $85 \%$ for three day and five day treatments, respectively. Loss to follow up was $5.4 \%$ by day 5 . There were no deaths, 41 hospitalisations, and 36 minor adverse reactions. There were 225 (10.3\%) clinical failures and $106(5.3 \%)$ relapses, and rates were similar in both treatments. At enrolment, 513 (23.4\%) children tested positive for respiratory syncytial virus, and Streptococcus pneumoniae and Haemophilus influenzae were isolated from the nasopharynx in $878(40.4 \%)$ and $496(22.8 \%)$ children, respectively. Clinical failure was associated with isolation of respiratory syncytial virus (adjusted odds ratio 1.95 (95\% confidence interval 1.0 to $3.8)$ ), excess respiratory rate of $>10$ breaths/minute (2.89 (1.83 to 4.55$))$, and non-adherence with treatment at day 5 (11.57 (7.4 to 18.0$)$ ).

Conclusions Treatment with oral amoxicillin for three days was as effective as for five days in children with non-severe pneumonia.
\end{abstract}

\section{Introduction}

Acute respiratory infections account for about 2.1 million deaths annually in children younger than 5 years. ${ }^{12}$ Since most cases of community acquired pneumonia are due to Haemophilus influenzae and Streptococcus pneumoniae, co-trimoxazole, penicillin, ampicillin, and amoxicillin have been recommended for control programmes. ${ }^{3}$ Since case management strategies were shown to be effective, ${ }^{4}$ India launched a control programme for acute respiratory infections, recommending the use of co-trimoxazole.

Despite evidence of rising bacterial resistance to co-trimoxazole, ${ }^{56}$ two recent studies reported good clinical efficacy of oral co-trimoxazole for non-severe pneumonia, ${ }^{78}$ although one reported that the failure rate for severe and radiologically proved pneumonia was twice that with amoxicillin treatment. ${ }^{8}$ Amoxicillin has been recommended as a suitable alternative because of its proved efficacy against $S$ pmeumoniae and H influenzae.

A trial of oral co-trimoxazole in Bangladeshi children reported that three days of treatment cured $75 \%$ of cases of nonsevere pneumonia with no subsequent treatment. ${ }^{9}$ A randomised controlled trial from Pakistan showed that three days and five days of treatment with oral amoxicillin had equivalent cure rates in children with non-severe pneumonia. ${ }^{10}$ To confirm these findings, we conducted the present study. Our primary hypothesis was that three days of treatment with oral amoxicillin is as effective as five days' treatment for non-severe pneumonia. Our secondary hypothesis was that relapse rates would be same in the two treatment regimens.

\section{Participants and methods}

This double blind, placebo controlled, randomised trial was conducted in the outpatient departments of seven referral hospitals in India. Participants were children aged 2-59 months with complaints of cough, rapid respiration, or difficulty in breathing. We defined non-severe pneumonia as a respiratory rate of $\geq 50$ breaths per minute (for ages $2-11$ months) or $\geq 40$ per minute (for age 12-59 months). We excluded children who had signs of severe pneumonia or disease (cyanosis, convulsions, inability to drink, difficult to wake, severe malnutrition, stridor), other conditions requiring antibiotic treatment, clinically recognised congenital heart disease, chronic systemic disorders, a history of repeated wheezing or asthma, been hospitalised in the previous two weeks, taken antibiotics in the previous two days, measles within the previous month, or a history of penicillin allergy and those already enrolled in the study.

Two stage screening was used (verbal followed by standardised screening) for inclusion and exclusion criteria. Patients with fever or wheeze received symptomatic treatment before enrolment. Those whose fast breathing persisted were enrolled after their parents or guardian had given their informed and written consent and randomised to either treatment and baseline data collected. 
The coordinating centre ensured quality assurance and provided standardised training for staff treating and assessing participants.

\section{Objectives}

Our primary objective was to compare the proportions of children recovering after three days' treatment and five days' treatment. Secondary objectives were to compare the proportions who relapsed within the next 6-14 days, the proportions who had resistant strains of $S$ pneumoniae or H influenzae in nasopharyngeal cultures at enrolment and at 14 day follow up, and the direct medical costs of treating clinical failures and relapses, and to assess the proportion of participants whose nasopharyngeal aspirates was positive for respiratory syncytial virus at enrolment.

\section{Intervention}

All participants received scored dispersible tablets of amoxicillin $125 \mathrm{mg}$ for the first three days. Amoxicillin was given thrice daily dissolved in $5 \mathrm{ml}$ of water, and the effective dose per kilogram body weight varied from 31 to $54 \mathrm{mg} /$ day. For the next two days participants received either amoxicillin or placebo.

\section{Sample size and randomisation}

We calculated sample size to test the equivalence hypothesis. Assuming a treatment failure rate of $12 \%$ with five day treatment, we would consider the two treatments to be equivalent if the failure rate with the shorter course of treatment was not more than $17 \%$ (that is, a difference of $5 \%$ or less). ${ }^{10}$ We were prepared to allow this size of difference, because there are other benefits of short course treatment, including lower cost and less frequent side effects.

We planned one interim analysis after a third of participants had been recruited. Using the O' Brien-Fleming approach, ${ }^{11}$ we carried out interim analysis at an $\alpha$ level of 0.005 and the final analysis at 0.047 . We used one sided testing, with power set at $90 \%$. The required number of patients was 950 in each group.

Block randomisation, with variable sized blocks, was done for each participating site to avoid unblinding. For both treatment groups, tablets were placed in serially numbered opaque white envelopes, each of which contained a green envelope containing 11 doses of amoxicillin for three days and a blue envelope containing eight doses of either amoxicillin or placebo for the next two days. Doses were provided for a maximum body weight of 20 $\mathrm{kg}$. Extra doses were provided for in case a child vomited within 30 minutes of taking treatment.

\section{Outcomes}

Participants were followed up at three and five days after enrolment and then again at between 12 and 14 days after enrolment. Home visits were done within 24 hours for those who failed to report on the appointed days. Mothers or care providers were asked to categorise their child as completely well, improved but still sick, the same, or worse at each follow up visit. Children who did not respond to treatment, developed adverse reactions to amoxicillin, or withdrew from the study were treated according to standard hospital guidelines.

Treatment failure was defined as development of chest indrawing, convulsions, drowsiness, or inability to drink at any time; respiratory rate above age specific cut-off points on day 3 or later; or oxygen saturation by pulse oximetry $<90 \%$ on day 3 . Participants who did not fail on assessment at day 3 or day 5 were considered clinically cured. Loss to follow up or withdrawal from the study at any time after recruitment was considered as treatment failure in our intention to treatment analysis. Relapse was defined as recurrence of signs of pneumonia or severe disease after day 5 among those who had been clinically cured at that time.

Treatment adherence was assessed by pill count on follow up days. Non-adherence was defined as intake of less than seven doses by day 3 and of less than five doses between days 3 and 5 .

Laboratory procedures-Presence of respiratory syncytial virus in nasopharyngeal aspirate was assessed at enrolment by means of a Becton Dickinson Directogen RSV kit. Nasopharyngeal swabs were taken at enrolment and at 14 days follow up to isolate $S$ pneumoniae and $H$ influenzae. Swabs were plated on 5\% sheep blood agar with gentamicin for $S$ pneumoniae and modified chocolate agar with bacitracin $(300 \mu \mathrm{g} / \mathrm{ml})$ for $H$ influenzae. Antibiotic susceptibility was tested by disc diffusion method as recommended by the National Committee for Clinical Laboratory Standards. ${ }^{12}$

Cost data collection-Participants who did not respond to treatment or who relapsed were followed to collect data on the medical resources they used. To calculate the costs of these resources, we listed the private sector hospitals that cater to lower middle class in each city of Lucknow, Vellore, Mumbai, New Delhi, Nagpur, Chandigarh, and Trivandrum and, by random draw, selected three hospitals in each city and obtained the prices of resources from them. We averaged the unit prices of all medical resources over the participating sites and used these averages in the analysis. The total estimated cost was the aggregated cost of drugs, investigations, hospitalisation, procedures and consultations, and out of pocket expenditures.

\section{Statistical analysis}

Clinical data analysis-Our primary analyses were done on an intention to treat basis. We calculated the difference in clinical cure rate (and 95\% confidence interval). We also performed per protocol analysis for participants with complete follow up and adherence to treatment. We compared baseline and other characteristics and therapeutic failures between the two treatment groups. We used the $\chi^{2}$ test for categorical variables and Student's $t$ test for continuous variables. We calculated crude odds ratios and 95\% confidence intervals. We constructed a multivariate model to assess determinants of treatment failure by forward stepwise logistic regression; the final model included covariates that were statistically significant. We analysed data using STATA, SPSS, and EP16.

Cost analysis -We analysed costs from the payer's perspective. We multiplied the units of each resource used by its average cost to calculate the total expenditure on that component of treatment. Univariate analysis was done to compare direct medical costs in the two treatment groups. We used Student's $t$ test to compare costs.

\section{Results}

We recruited 2188 patients from August 2000 to December 2002 and randomised 1095 to three days of amoxicillin treatment and 1093 to five days of treatment (figure). Loss to follow up was $5.4 \%$ by day 5 , and $6.8 \%$ by day 14 . There were no substantial differences in the baseline characteristics of the treatment groups (table 1).

\section{Adherence to treatment}

Table 1 shows adherence to treatment. The mean doses taken from the green and blue envelopes were 8.9 (SD 0.9) out of nine doses and 5.56 (SD 1.6) out of six doses, respectively, and were similar in both the groups. 


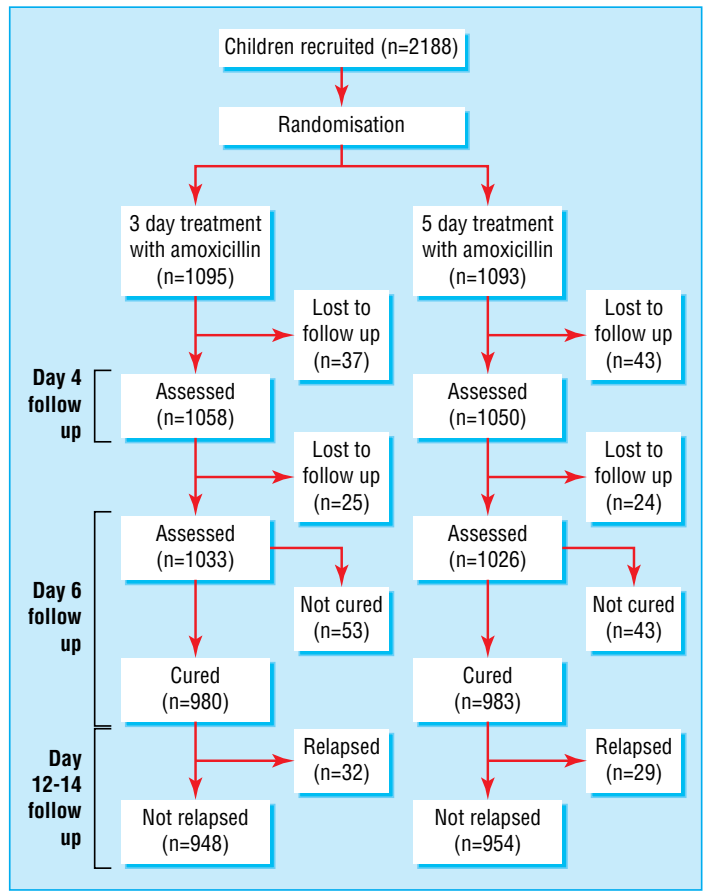

Flow of participants through each stage of the randomised trial

\section{Primary and secondary clinical outcomes}

In our intention to treat analysis, clinical cure rates were $89.5 \%$ $(980 / 1095)$ and $89.9 \%(983 / 1093)$ in the three day treatment and five day treatment groups, respectively (table 2), similar among wheezers and non-wheezers. In the per protocol analysis, the clinical cure rates were $94.9 \%(980 / 1033)$ and $95.8 \%$ (983/1026). There was also no difference between groups in the rate of relapse among those considered cured on day 5 (table 2).

\section{Microbiology outcomes}

A total of $513(23.4 \%)$ patients tested positive for respiratory syncytial virus at enrolment (table 1), of whom $8.7 \%$ had wheeze. Table 3 shows the antimicrobial resistance pattern for the 878 isolates of S pneumoniae and 496 isolates of $H$ influenzae cultured at enrolment. On day 14, isolation rates of $S$ pneumoniae and $H$ influenzae were $10.9 \%(\mathrm{n}=325)$ and $6.9 \%(\mathrm{n}=249)$, respectively, and did not differ by treatment type (table 3 ). While there was no change in resistance of $H$ influenzae over time, the proportion of $S$ pneumoniae isolates resistant to co-trimoxazole rose significantly from $66.1 \%$ to $78.2 \%(\mathrm{P}=0.02)$ over 15 days in the five day amoxicillin treatment group. We found no increase in emergence of antimicrobial resistance in $S$ pneumoniae or $H$ influenzae in individual patients, based on a paired analysis (data not shown).

\section{Risk factors associated with clinical failure}

Table 4 shows the univariate associations of clinical failure with baseline variables. In logistic regression, clinical failure was significantly associated with non-adherence at day 5 (adjusted odds ratio 11.57 (95\% confidence interval 7.4 to 18.0$)$ ), and excess respiratory rate of $>10$ breaths/minute (2.89 (1.83 to $4.55)$ ), and nasopharyngeal swab positivity for respiratory syncytial virus (1.95 (1.0 to 3.8$))$.

\section{Association of clinical cure with caregivers' assessment}

Of the 1963 patients assessed as clinically cured, mothers or carers reported that $1005(51.2 \%)$ were completely well, $938(47.8 \%)$ were improved but still sick, 26 (1.3\%) were the same, and one
Table 1 Baseline characteristics of 2188 children with non-severe pneumonia randomised to 3 days or 5 days of treatment with amoxicillin. Values are numbers (percentages) of patients unless stated otherwise

\begin{tabular}{|c|c|c|}
\hline Characteristic & 3 day treatment $(n=1095)$ & 5 day treatment $(n=1093)$ \\
\hline Mean (SD) age (months) & $17.0(13.3)$ & $16.9(13.0)$ \\
\hline Mean (SD) height (cm) & $74.8(10.98)$ & $74.8(10.75)$ \\
\hline Mean (SD) weight (kg) & 8.7 (2.49) & $8.7(2.4)$ \\
\hline $\begin{array}{l}\text { Mean }(\mathrm{SD}) \text { duration of illness } \\
\text { (days) }\end{array}$ & $4.7 \quad(3.43)$ & $4.5 \quad(3.12)$ \\
\hline Mean (SD) temperature $\left({ }^{\circ} \mathrm{C}\right)$ & $37.1 \quad(0.66)$ & $37.2(0.67)$ \\
\hline \multicolumn{3}{|l|}{$\begin{array}{l}\text { Mean (SD) respiratory rate } \\
\text { (breaths/minute): }\end{array}$} \\
\hline 2-11 months old & $56.4 \quad(5.02)$ & $56.0(4.54)$ \\
\hline 12-59 months old & $47.3(5.58)$ & $47.9(6.1)$ \\
\hline Male & $685 \quad(62.6)$ & $\begin{array}{ll}676 & (61.8)\end{array}$ \\
\hline \multicolumn{3}{|l|}{ Age (months): } \\
\hline $2-11$ & $479 \quad(43.7)$ & $475 \quad(43.5)$ \\
\hline $12-59$ & $\begin{array}{ll}616 & (56.3)\end{array}$ & $618 \quad(56.5)$ \\
\hline \multicolumn{3}{|l|}{ Weight for height z score*: } \\
\hline-2 to -1 & $300 \quad(27.4)$ & $303 \quad(27.7)$ \\
\hline-3 to -2 & $188 \quad(17.2)$ & $183 \quad(16.7)$ \\
\hline \multicolumn{3}{|l|}{ Duration of illness (days): } \\
\hline$<3$ & $538 \quad(49.1)$ & $540 \quad(49.4)$ \\
\hline$\geq 3$ & $557 \quad(50.9)$ & $553 \quad(50.6)$ \\
\hline Fever & $833 \quad(76.1)$ & $850 \quad(77.8)$ \\
\hline Cough & 1081 (98.7) & $1078 \quad(98.6)$ \\
\hline Difficulty in breathing & $417 \quad(38.1)$ & $387 \quad(35.4)$ \\
\hline Vomiting & $135 \quad(12.3)$ & $141 \quad(12.9)$ \\
\hline Diarrhoea & $71 \quad(6.5)$ & $55 \quad(5.0)$ \\
\hline \multicolumn{3}{|l|}{$\begin{array}{l}\text { Excess respiratory rate } \\
\text { (breaths/minute)t: }\end{array}$} \\
\hline$\leq 10$ & $903 \quad(82.5)$ & $881 \quad(80.6)$ \\
\hline$>10$ & $192 \quad(17.5)$ & $212 \quad(19.4)$ \\
\hline Wheeze present & $140 \quad(12.8)$ & $147 \quad(13.4)$ \\
\hline \multicolumn{3}{|l|}{ Adherence to treatment: } \\
\hline At day 3 & $1031 \quad(94.2)$ & $1026 \quad(93.9)$ \\
\hline At day 5 & $937 \quad(85.6)$ & $928 \quad(84.9)$ \\
\hline RSV positive & 252 (23.0) & 261 (23.9) \\
\hline
\end{tabular}

*Z score given as number of standard deviations from normal value. †Rate above the age specific cut off. $\mathrm{RSV}=$ respiratory syncytial virus.

$(0.1 \%)$ was worse. Of the 96 patients assessed as not cured, mothers reported that $4.2 \%$ were completely well, $63.5 \%$ were improved but still sick, $29.2 \%$ were the same, and 3.1\% were worse $(\mathrm{P}=0.001)$.

\section{Cost analysis}

Mean annual family income was 3248.5 rupees (SD 3175.29). Average direct medical costs of successful treatment with amoxi-

Table 2 Comparison of outcome measures in 2188 children with non-severe pneumonia randomised to 3 days or 5 days of treatment with amoxicillin: intention to treat analysis. Values are numbers (percentages) of patients unless stated otherwise

\begin{tabular}{lccc} 
& $\begin{array}{c}\mathbf{3} \text { day treatment } \\
(\mathbf{n = 1 0 9 5 )}\end{array}$ & $\begin{array}{c}\mathbf{5} \text { day treatment } \\
(\mathbf{n = 1 0 9 3 )}\end{array}$ & Difference (95\% Cl) \\
\hline $\begin{array}{l}\text { Primary outcome } \\
\text { measures: }\end{array}$ & & & \\
\hline Cure on day 5 & $980(89.5)$ & $983(89.9)$ & $0.4(-2.1$ to 3.0) \\
\hline Relapse after day 5 & $58(5.3)$ & $48(4.4)$ & $1.0(-1.0$ to 3.0) \\
\hline $\begin{array}{l}\text { Secondary outcome } \\
\text { measure: }\end{array}$ & & & \\
\hline $\begin{array}{l}\text { Cure on day 5 } \\
\text { among wheezers }\end{array}$ & $127 / 140(90.7)$ & $132 / 147(89.8)$ & $0.9(-5.9$ to 7.8$)$ \\
\hline $\begin{array}{l}\text { Cure on day } 5 \\
\text { among } \\
\text { non-wheezers }\end{array}$ & $853 / 957(89.1)$ & $851 / 946(90.0)$ & $0.7(-2.1$ to 3.4) \\
\hline
\end{tabular}


Table 3 Antimicrobial resistance of isolates of Streptococcus pneumoniae and Haemophilus influenzae from children with non-severe pneumonia randomised to 3 days or 5 days of treatment with amoxicillin. Isolates cultured from nasopharyngeal swabs taken at enrolment (day 0 ) and final follow up (day 14 )

\begin{tabular}{|c|c|c|c|c|c|c|}
\hline \multirow[b]{3}{*}{ Antibiotic resistance* } & \multicolumn{4}{|c|}{ No of resistant isolates/total tested (\%) } & \multicolumn{2}{|c|}{$\begin{array}{c}\text { P value of difference between } \\
\text { treatments }\end{array}$} \\
\hline & \multicolumn{2}{|c|}{ Day 0} & \multicolumn{2}{|c|}{ Day 14} & \multirow{2}{*}{\multicolumn{2}{|c|}{$\begin{array}{cc}\text { Change from day } \\
\text { At day } 14 \quad 0 \text { to day } 14\end{array}$}} \\
\hline & 3 day treatment & 5 day treatment & 3 day treatment & 5 day treatment & & \\
\hline \multicolumn{7}{|l|}{ S pneumoniae: } \\
\hline Co-trimoxazole & $253 / 380(66.6)$ & $252 / 381(66.1)$ & $106 / 159(66.7)$ & $111 / 142(78.2)$ & 0.02 & 0.05 \\
\hline Chloramphenicol & $21 / 418 \quad(5.0)$ & $14 / 419$ (3.3) & 9/163 (5.5) & $6 / 142 \quad(4.2)$ & 0.6 & 0.89 \\
\hline Oxacillin & $67 / 408$ (16.4) & $64 / 413(15.5)$ & $17 / 160(10.6)$ & $17 / 141(12.1)$ & 0.7 & 0.59 \\
\hline Erythromycin & $15 / 421 \quad(3.6)$ & $9 / 418 \quad(2.2)$ & $2 / 161 \quad(1.2)$ & $4 / 142 \quad(2.8)$ & 0.3 & 0.14 \\
\hline \multicolumn{7}{|l|}{ H influenzae: } \\
\hline Co-trimoxazole & $118 / 217(54.4)$ & $133 / 218(61.0)$ & $74 / 129(57.4)$ & $64 / 106(60.4)$ & 0.64 & 0.65 \\
\hline Chloramphenicol & $58 / 232(25.0)$ & $57 / 234(24.4)$ & $27 / 126(21.4)$ & $24 / 108(22.2)$ & 0.88 & 0.83 \\
\hline Erythromycin & $69 / 230(30.0)$ & $65 / 232(28.0)$ & $39 / 126(31.0)$ & $31 / 108(28.7)$ & 0.7 & 0.97 \\
\hline Ampicillin & $46 / 235$ (19.6) & $40 / 237$ (16.9) & $30 / 129(23.3)$ & $24 / 108(22.2)$ & 0.85 & 0.8 \\
\hline
\end{tabular}

*Resistance based on zone of inhibition in $\mathrm{mm}$. $S$ pneumoniae resistant to oxacillin $(<20)$, chloramphenicol $(\leq 20)$, erythromycin $(\leq 15)$, co-trimoxazole $(\leq 15)$. $H$ influenzae resistant to ampicillin $(<18)$, chloramphenicol $(<25)$, erythromycin $(<15)$, co-trimoxazole $(<10)$.

cillin for three days and five days were 11 and 19 rupees, respectively. Cost data were available for most cases of treatment failure $(\mathrm{n}=183,82.03 \%)$ and relapse $(\mathrm{n}=84,79.2 \%)$. The mean direct medical cost of treating those who had not responded to treatment or had relapsed was 272.79 rupees (SD 514.2) in both treatment groups. From our study data, we calculate that the average direct medical costs of treating 1000 cases of non-severe pneumonia with three days or fives days of amoxicillin would be 54930 rupees (£790, \$1100) and 62430 rupees (£900, $\$ 1250)$, respectively. Among those who had not responded to treatment or had relapsed, $109(40.8 \%)$ received bronchodilators with or without corticosteroids, $265(99.2 \%)$ received antibiotics other than amoxicillin, 65 (24.3\%) had chest radiographs, 11 required an intravenous line, two underwent continuous pulse oximetry, and one required intercostal drainage.

\section{Adverse reactions}

Adverse reactions were similar in both treatment arms. There were no deaths, purpura, or serious adverse effects of amoxicillin. There were 41 hospitalisations, with similar numbers in the three day and five day treatments (18 and 23, respectively). There were

Table 4 Association of baseline characteristics with clinical cure or treatment failure among 2188 children with non-severe pneumonia randomised to 3 days or 5 days treatment with amoxicillin: intention to treat analysis. Values are numbers (percentages) of patients unless stated otherwise

\begin{tabular}{|c|c|c|c|}
\hline Characteristic & Cure $(n=1963)$ & $\begin{array}{l}\text { Treatment failure } \\
\quad(n=225)\end{array}$ & Odds ratio $(95 \% \mathrm{Cl})$ \\
\hline Breathing difficulty & $726(37.0)$ & 78 (34.7) & $1.1 \quad(0.8$ to 1.5$)$ \\
\hline Cough & 1938 (98.7) & $221(98.2)$ & 1.4 (0.5 to 4.1$)$ \\
\hline Fever & $1518(77.3)$ & $165(73.3)$ & 1.2 (0.9 to 1.7$)$ \\
\hline Vomiting & $246(12.5)$ & $30(13.3)$ & $0.9 \quad(0.6$ to 1.4$)$ \\
\hline Age $<12$ months & $860(43.8)$ & $94(41.8)$ & $1.1 \quad(0.8$ to 1.4$)$ \\
\hline Male & $1220(62.1)$ & $141(62.7)$ & $1.0 \quad(0.8$ to 1.4$)$ \\
\hline Wheeze & $259(13.2)$ & $28(12.4)$ & 1.1 (0.7 to 1.6$)$ \\
\hline $\begin{array}{l}\text { Duration of illness } \leq 3 \\
\text { days }\end{array}$ & $959(48.9)$ & $119(52.9)$ & $0.8 \quad(0.6$ to 1.1$)$ \\
\hline $\begin{array}{c}\text { Excess respiratory rate } \\
<10 \text { breaths/minute }\end{array}$ & 1627 (82.9) & $157(49.8)$ & $2.1 \quad$ (1.5 to 2.9$)$ \\
\hline $\begin{array}{c}\text { Excess respiratory rate } \\
<6 \text { breaths/minute }\end{array}$ & $1183(60.3)$ & $108(48.0)$ & $1.6 \quad(1.2$ to 2.2$)$ \\
\hline RSV positive & $448(22.8)$ & $65(28.9)$ & $0.7 \quad(0.5$ to 0.99$)$ \\
\hline $\begin{array}{l}\text { Weight for height } z \\
\text { score }-3 \text { to }-2\end{array}$ & $339(17.3)$ & $32(14.2)$ & $1.3 \quad(0.9$ to 1.9$)$ \\
\hline $\begin{array}{l}\text { Adherence to } \\
\text { treatment at day } 5\end{array}$ & 1818 (92.6) & $47(20.9)$ & 47.5 (33.0 to 68.3 ) \\
\hline
\end{tabular}

$\mathrm{RSV}=$ respiratory syncytial virus. three cases of severe vomiting, 20 cases of diarrhoea with some dehydration, four cases of rash without itch, once case of rash with itch, and eight cases of wheezing in a child without wheeze at enrolment.

\section{Discussion}

We found that treatment with oral amoxicillin for either three days or five days was equally effective for non-severe pneumonia. Among children with complete follow up who adhered to treatment, cure rate was about $95 \%$. From the numbers needed to treat, we calculate that 250 cases of non-severe pneumonia would need to be treated with five days of amoxicillin rather than three days for one additional cure.

Amoxicillin is a bactericidal drug and is effective against $S$ pneumoniae and $H$ influenzae. Short courses of amoxicillin have been used to treat infections caused by these and other organisms causing tonsillo-pharyngitis, ${ }^{13}$ urinary tract infections ${ }^{14}$ and other common childhood infections. ${ }^{15}$ Hence, it is rational to expect that amoxicillin would work in shorter duration. In addition, equivalence of three and five day treatment with amoxicillin for non-severe pneumonia has also been reported in a study from Pakistan. ${ }^{10}$

\section{Strengths and limitations of study}

The main strengths of our trial were that it was large, double blind, and multicentre and was conducted over two years covering all four seasons with a minimal loss to follow up and good adherence to treatment. Its limitations are that is was a hospital based study, causes of infection were not investigated, follow up was limited to only 15 days, and children with history of asthma were excluded.

\section{Risk factors for treatment failure}

Risk factors associated with treatment failure in our study were an excess respiratory rate of more than 10 breaths per minute above the age specific cut off, non-adherence to treatment at day 5 , and nasopharyngeal swab positivity for respiratory syncytial virus. Unlike in the Pakistan study, ${ }^{10}$ we did not find any difference of outcomes in children aged $<12$ months compared with older children. Possible explanations may be the lower proportion of infants recruited by us and variation between our study sites. Since almost half of the children's mothers or carers did not agree with a doctor's assessment of cure in our study, parents may need appropriate counselling or else may seek treatment elsewhere. 


\section{What is already known on this topic}

Amoxicillin is effective treating non-severe pneumonia in children

Isolation of infective bacteria from the nasopharynx can be used to monitor antimicrobial resistance in the community

\section{What this study adds}

Three days of treatment with amoxicillin is as effective as the standard five day course in treating non-severe pneumonia

Almost three quarters of nasopharyngeal isolates of Streptococcus pneumoniae and Haemophilus influenzae were resistant to co-trimoxazole

Respiratory syncytial virus, the commonest viral cause of pneumonia, may lead to severe disease, particularly in young children. ${ }^{16}{ }^{17}$ In our study, nasopharyngeal swabbing of $23 \%$ of patients tested positive for the virus. Because we excluded patients with severe disease, we possibly missed many other infected children. ${ }^{16}$ Viral detection technique is also of prime importance; the detection kit used in our study had a sensitivity of $83 \%{ }^{18}$ Detection of the virus in our study increased the probability of treatment failure. We did not find an association between the virus and wheezing, unlike others. ${ }^{19}$

$S$ pneumoniae and $H$ influenzae are the commonest bacterial agents of pneumonia in children. ${ }^{19}$ As in other studies, ${ }^{20}$ our carrier rate for either bacteria at enrolment was less than $50 \%$. We found a significant rise in resistance of $S$ pneumoniae to co-trimoxazole from enrolment until day 14 in children receiving five days of treatment, as has been reported elsewhere. ${ }^{21}$ In many regions resistance to only one antibiotic is gradually replaced by resistance to two or more antibiotic classes. ${ }^{22}$ This occurs because the bacterial serotypes that are carried most often by people are commonly also carried for prolonged periods and are thus exposed to multiple antibiotics. Therefore, resistance to various antibiotic classes with different mechanisms may occur in the same strain, giving this strain biological advantages in the selection process. This phenomenon results in the use of one class of antibiotics promoting carriage of $S$ pneumoniae resistant to another antibiotic class. ${ }^{23}$

\section{Conclusions}

We recommend the three day course of amoxicillin for treating community acquired non-severe pneumonia in children, as this is equally as effective as a five day course but is cheaper with increased adherence and possibly decreased emergence of antimicrobial resistance. Our findings have local as well as global implications, because our study has also confirmed findings from a recently published data from elsewhere.

Thomas Cherian assisted in protocol development and supervised initial data collection from Vellore. Robert and Suzanne Fletcher assisted in manuscript development under IndiaClen-CBS workshop.

Shally Awasthi was the project coordinator and is guarantor for the study. Members of the ISCAP Study Group are listed below (in alphabetical order). Clinical investigators: Shally Awasthi, King George's Medical University, Lucknow, India; S K Kabra, All India Institute of Medical Sciences, New Delhi, India; Madhuri Kulkarni, Lok Manya Tilak Medical College and Hospital, Mumbai, India; N Murali, Christian Medical College, Vellore, India; A K Niswade, Government Medical College, Nagpur, India; R M Pillai, Government Medical College, Trivandrum, India; Sunit Singhi, Postgraduate Institute of Medical Sciences, Chandigarh, India. Microbiology: Chhaya A
Chande, Government Medical College, Nagpur; Bimal Das, All India Institute of Medical Sciences, New Delhi; Amita Jain, King George's Medical University, Lucknow; Jyoti Kamath and Meenakshi Mathur, Lok Manya Tilak Medical College and Hospital, Mumbai; Kavitha Raje, Government Medical College, Trivandrum; Pranab Roy, Postgraduate Institute of Medical Sciences, Chandigarh; M K Lalitha, Christian Medical College, Vellore. Biostatistician: Girdhar Agarwal, Lucknow University, Lucknow. Randomisation: L Jayseelan, Christian Medical College, Vellore. Technical adviser: Shamim Qazi, medical officer, Department of Child and Adolescent Health and Development (CAH),WHO, Geneva, contributed to development of the protocol, provided technical assistance during the trial, and helped write the manuscript. Manuscript writing committee: Girdhar Agarwal, Lucknow; Shally Awasthi, Lucknow; S K Kabra, New Delhi; Annapurna Kaul, King George's Medical University, Lucknow; Sunit Singhi, Chandigarh; Stephen D Walter, McMaster University, Canada. Data monitoring safety committee: J N Pande, chair, All India Institute of Medical Sciences, New Delhi; Stephen D Walter, McMaster University; Indu Wakhlu, King George's Medical University, Lucknow.

Funding: US AID through INCLEN and IndiaClen.

Competing interests: None declared.

Ethical approval: The ethics committees of the participating institutions approved the study.

1 Mathers CD, Murray CJL, Lopez AD, Stein C. The global burden of disease 2000 project: objectives, methods, data sources and preliminary results. Evidence and information for policy (EIP). Geneva: World Health Organization, 2001

2 World Health Organization. World health report 1999:making a difference. Geneva: WHO, 1999.

3 WHO memorandum. Clinical management of acute respiratory infections in children. Bull World Health Organ 1981;59:707-16.

4 Sazawal S, Black ER. Pneumonia case management trials group. Effect of pneumonia case management on mortality in neonates, infants and preschool children. A meta-analysis of community based trials. Lancet Infect Dis 2003;3:547-56.

5 Invasive Bacterial infection Surveillance (IBIS) Group, INCLEN. Prospective multicentric hospital surveillance of Streptococcus pneumoniae disease in India. Lancet tric hospital surve

6 Timothy D, Mastro MD, Nomani N, Ishaq Z, Ghafoor A, Shaukat NF, et al. Use of nasopharyngeal isolates of Streptococcus pneumoniae and Haemophilus influenzae from children in Pakistan for surveillance for antimicrobial resistance. Pediatr Infect Dis J 1993; 12:824-30.

7 Rasmussen Z, Bari Z, Qazi SA. Standard versus double strength co-trimoxazole for the treatment of childhood pneumonia: a double blind, randomized multicentric trial in Pakistan. International Journal of Tuberculosis and Lung Diseases 1997;1:119.

8 Straus WL, Qazi S, Kundi Z, Normani NK, Schwartz B. Antimicrobial resistance and clinical effectiveness of co-trimoxazole versus amoxicillin for pneumonia among children in Pakistan: randomized controlled trial. Lancet 1998;352:270-4.

9 DeFransico A, Chakraborty J. Adherence to co-trimoxazole treatment for lower respiratory tract infection in rural Bangladeshi children. Ann Trop Pediatr 1998;18:17-21.

10 MASCOT group. Clinical efficacy of three days versus five days of oral amoxicillin for the treatment of childhood pneumonia: a multicentre double-blind trial. Lancet 2002;360:835-41.

11 O'Brien PC, Fleming TR. A multiple testing procedure for clinical trials. Biometrics 1979;35:549-56.

12 NCCLS (National Committee for Clinical Laboratory Standards). www.nccls.org (accessed 7 Mar 2004).

13 Pichichero ME, Cohen R. Shortened course of antibiotic therapy for acute otitis media, sinusitis and tonsillopharyngitis. Pediatr Infect Dis J 1997;16:680-95.

14 Michael M, Hodson EM, Craig JC, Martin S, Moyer VA. Short versus double duration oral antibiotic therapy for acute urinary tract infection in children. Cochrane Database Syst Rev 2003;1:CD003966.

15 Peltola H, Vuori-Holopainen E, Kallio MJ, SE-TU Study Group. Successful shortening from seven to four days of parenteral beta-lactam treatment for common childhood infections: a prospective and randomized study. Int J Infect Dis 2001;5:3-8.

16 Parkin PC, Taylor CY, Petric M, Schuh S, Goldbach M, Ipp M. Controlled study of respiratory viruses and wheezing. Arch Dis Child 2002;87:221-2.

17 Weber MW, Mulholland EK, Greenwood BM. Respiratory synctial virus infection in tropical and developing countries. Trop Med Int Health 1998:3:268-80.

18 Kok T, Barancek K, Burrell CJ. Evaluation of the Becton Dickinson Directigen for respiratory synctial virus in nasopharyngeal aspirates. Arch Pathol Lab Med piratory synctial
$1991 ; 115: 451-8$.

19 Shann F. Etiology of severe pneumonia in children in developing countries. Pediatr Infect Dis 1986;4:247-52

20 Varon E, Levy C, Rocque FDL, Boucherat M, Deforche D, Podglajen I, et al. Impact of antimicrobial therapy on nasopharyngeal carriage of Streptococcus pneumoniae, Haemophilus influenzae and Branhamella catarrhalis in children with respiratory tract infections. Clin Infect Dis 2000;31:477-81.

21 Schrag SJ, Pena C, Fernandez J, Sanchez J, Gomej V, Perez E, et al. Effect of short course, high dose amoxycillin therapy on resistant pneumococcal carriage. JAMA 2001;286:49-56

22 McCormick AW, Whitney CG, Farley MM, Lynfield R, Harrison LH, Bennett NM, et al Geographic diversity and temporal trends of antimicrobial resistance in Streptococcus pneumoniae in the United States. Nat Med 2003;9:424-30.

23 Arason VA, Kristinsson KG, Sigurdsson JA, Stefansdottir G, Molstad S, Gudmundsson $\mathrm{S}$. Do antimicrobials increase the carriage rate of penicillin resistant pneumococci in children? Cross sectional prevalence study. BMJ 1996;313:387-91.

doi 10.1136/bmj.38049.490255.DE 


\section{Amendment}

This is Version 2 of the paper. In this version, the clinical cure rates in the results section of the abstract are given as percentages [in the previous version the actual numbers were given].
Correspondence to: Professor Shally Awasthi, Department of Paediatrics and Clinical Epidemiology, King George's Medical University, Lucknow (UP)-226003, India sawasthi@sancharnet.in 